\title{
Phase 0 clinical trials: theoretical and practical implications in oncologic drug development
}

This article was published in the following Dove Press journal:

Open Access Journal of Clinical Trials

16 October 2013

Number of times this article has been viewed

\section{Preciosa M Coloma}

Department of Medical Informatics, Erasmus MC University Medical Center, Rotterdam, The Netherlands
Correspondence: Preciosa M Coloma Department of Medical Informatics, Erasmus MC University Medical Center, Postbus 2040, 3000 CA Rotterdam, The Netherlands $\mathrm{Tel}+3 \mathrm{I} 107044128$ Fax +3। 107044722 Email p.coloma@erasmusmc.nl
Abstract: Drug discovery and development has become a risky, expensive, and protracted process, with the cost of introducing a new drug to the market going as high as US\$2 billion and the entire process taking at least 10-15 years. Great advances in biomedical research in recent years have not resulted in translation into medical product development, and there has been substantial decline in both new drug applications and biological license applications. To address this so-called "pipeline problem," both the US Food and Drug Administration and its European counterpart, the European Agency for the Evaluation of Medicinal Products (now European Medicines Agency) endorsed the concept of Phase 0 studies (also known as exploratory investigational new drug studies), aimed towards identifying, early in the process of drug development, viable candidates and eliminating those lacking promise. Primary study endpoints of trials conducted under an exploratory investigational new drug can include evaluation of analogs for lead selection, modulation of a molecular target in vivo, whole-body imaging for tissue distribution/target binding affinity, and agent pharmacokinetics. Phase 0 trials bridge the gap between traditional preclinical testing and clinical studies and are intended to provide a better understanding of a new compound's pharmacokinetics, pharmacodynamics, and target localization before initiation of Phase I trials. When such information can be obtained earlier, decisions regarding drug development can also be made at an earlier point in time, potentially reducing costs of initial preclinical studies and time-to-first-in-human testing. This review provides an overview of the various conditions that have to be met in order for a Phase 0 trial to be successful, citing examples of two candidate drugs that have been further developed after Phase 0 trials in oncology. Challenges and opportunities with Phase 0 trials are discussed, including ethical issues associated with trials that have no therapeutic or diagnostic intent.

Keywords: pre-Phase I studies, exploratory IND, microdosing, oncology, cancer drug development

\section{Background}

The existing paradigm of drug discovery and development has become such an expensive and protracted process: the average cost of introducing a new drug to the market, including the cost of failures, has been estimated to be between US\$800 million and US\$2 billion. ${ }^{1-3}$ The entire process usually takes at least $10-15$ years. For anticancer drugs in particular, the failure rate is around $90 \% .{ }^{4}$ Promising candidate agents undergo a series of testing, initially in vitro using models that permit evaluation of receptor binding, effects on enzyme activities, toxic effects, and other in-vitro pharmacologic parameters (see Figure 1). Candidates that are not rejected during these early investigations subsequently undergo in-vivo testing for efficacy and safety. Further efficacy 


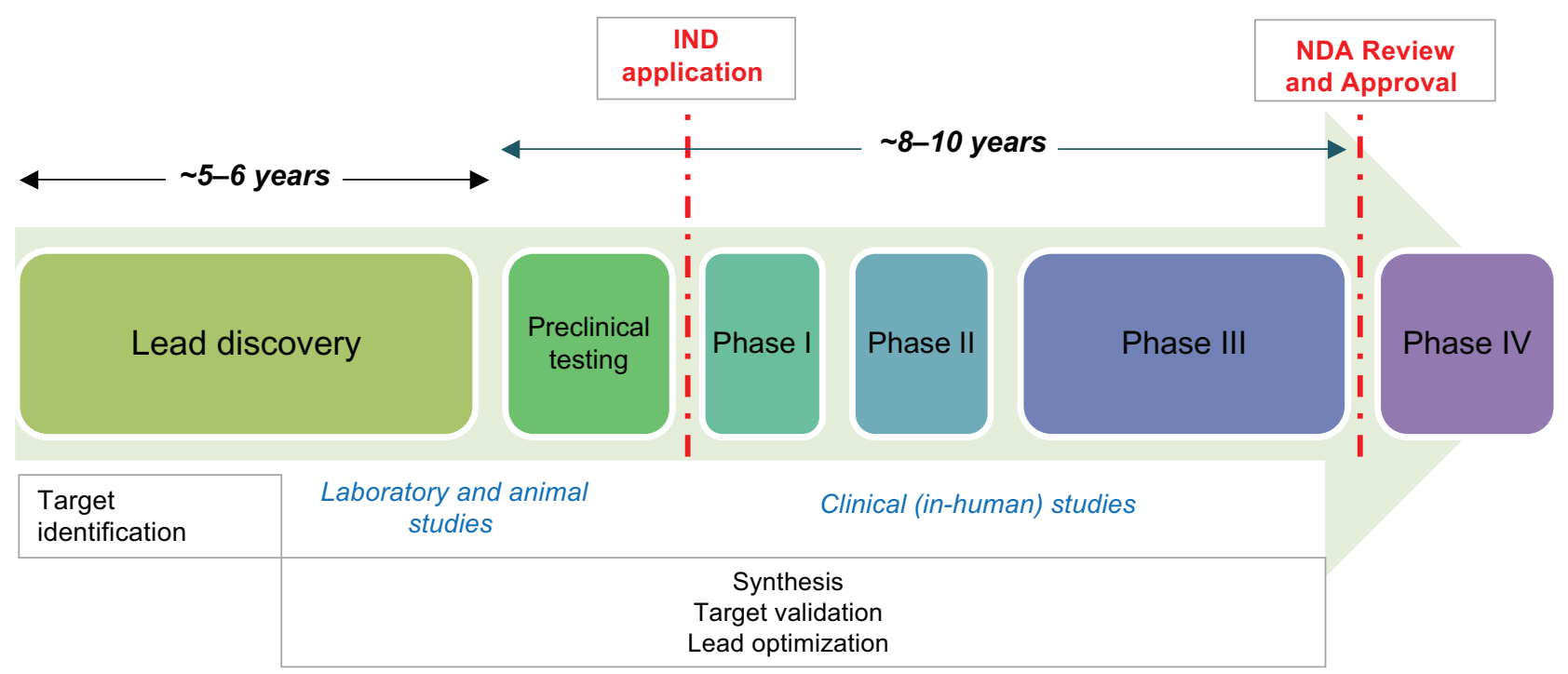

Figure I Overview of the traditional process of new drug development.

Notes: During new drug development, large numbers of compounds are generated with the aim of identifying the most promising candidates for further development. Promising candidates, generally structurally related, are often selected using in-vitro testing models that examine binding to receptors, effects on enzyme activities, or toxic effects. Candidates not rejected in these initial assessments subsequently undergo testing for efficacy and safety in animals, usually in rats and dogs. These animal studies are designed to permit selection of a safe starting dose for humans (including estimation of the margins of safety between clinical and toxic dose), to predict pharmacokinetic/ pharmacodynamic parameters, and to gain an insight as to which organs may be the subject of toxicity. Animal studies can provide substantial evidence of product effectiveness under certain circumstances.

Abbreviations: IND, investigational new drug; NDA, new drug application.

testing can be carried out in animals, and animal studies can provide substantial evidence of product effectiveness under the following circumstances. ${ }^{5}$

1. There is a reasonably well understood mechanism for the toxicity of the agent and its amelioration or prevention by the product.

2. The effect is demonstrated in more than one animal species expected to react with a response predictive for humans, unless the effect is demonstrated in a single animal species that represents a sufficiently well characterized animal model for predicting the response in humans.

3. The animal study endpoint is clearly related to the desired benefit in humans, generally the enhancement of survival or prevention of major morbidity.

4. The data or information on the kinetics and pharmacodynamics (PDs) of the product or other relevant data or information, in animals and humans, allows selection of an effective dose in humans.

Pharmacokinetic (PK) and PD data obtained from animal models may not always be predictive of human PKs/PDs, which makes the early phase of drug development tricky, risky, and expensive: this largely determines whether a new molecular entity is "druggable," ie, how likely it is able to modulate a target. ${ }^{6}$

\section{Exploratory investigational new drug (IND) studies}

Despite the huge advances in biomedical research worldwide, translation into medical product development has not been forthcoming. Recognizing this gap, the United States Food and Drug Administration (FDA) in its 2004 Critical Path Report: Innovation or Stagnation ${ }^{7}$ lamented the substantial decline of new drug applications and biologic license applications submitted to the agency and sought to address this so-called "pipeline problem." As a follow-through to this imperative to provide better tools and an insightful knowledge base to make drug development more efficient, the US FDA issued, in 2006, a Guidance on Exploratory IND Studies. ${ }^{8}$ Developed in conjunction with the pharmaceutical industry and the National Cancer Institute, this Guidance pointed out the existence of exploratory approaches consistent with regulatory requirements that maintain human subject protection while involving fewer resources than is usual with traditional early Phase trials. Investigators and sponsors have apparently underutilized these, but the US FDA believed such approaches could streamline the development of promising candidates. In a similar move earlier in 2003, the European Agency for the Evaluation of Medicinal Products (now European Medicines Agency [EMA]) released a position paper on the nonclinical safety studies needed to support human clinical 
trials with a single dose of a pharmacologically active compound using microdose techniques (CPMP/SWP/2599/02/). A related concept paper released by EMA later in 2006 recommended the drafting of a guidance document detailing what nonclinical data are required to be included in a clinical trials application for an early Phase I study in humans. This guideline was intended to allow for flexibility of approaches, including those outlined in the EU Microdose guideline or the US FDA exploratory IND guideline. ${ }^{9}$ Whereas the US FDA's guidance did not constitute a new regulation (it was presented as an interpretation of existing recommendations on drug development), EMA's position paper introduced the possibility of a reduced preclinical safety package for subpharmacological (micro) dose clinical studies. As described by EMA, microdose studies can be valuable in the evaluation of human plasma PKs as well as receptor selectivity profile of candidate drugs as early as possible in the preclinical stage of development. Theoretically, microdose trials could bring about an early decision with respect to distinguishing between promising and inappropriate molecules for further development. (This EMA position paper was later superseded by the ICH [International Conference on Harmonisation of Technical Requirements for Registration of Pharmaceuticals for Human Use] guideline M3[R2]). ${ }^{10}$

Exploratory IND studies refer to clinical trials that involve very limited human exposure and have no therapeutic or diagnostic intent. ${ }^{8}$ The preclinical pharmacology and toxicology testing required for an exploratory IND is less extensive than that for a traditional IND, and there are differences in the preclinical and clinical study pathways for traditional and exploratory IND applications. Primary study endpoints of trials conducted under an exploratory IND can include: ${ }^{11} 1$ ) evaluation of analogs for lead selection; 2) verification as to whether a mechanism of action defined in experimental systems can also be observed in humans (eg, a binding property or inhibition of an enzyme); 3) wholebody imaging for tissue distribution and target binding affinity; and 4) agent PKs.

\section{Phase 0 trials}

Because exploratory IND studies refer to clinical trials that are conducted early in Phase I, such studies have been subsequently dubbed "pre-Phase I studies" or "Phase 0 trials." Phase 0 trials bridge the gap between traditional preclinical testing and clinical studies and are intended to provide a better understanding of a new compound's PKs, PDs, and target localization before initiation of Phase I trials. When such information can be obtained earlier, decisions regarding drug development can also be made at an earlier point in time. ${ }^{12}$ Thus, Phase 0 trials, when optimized, may reduce costs of initial preclinical studies and time-to-first-in-human testing.

\section{Phase 0 and Phase I - what's the difference?}

Phase 0 trials are not intended to impart evidence of efficacy; neither should they be seen as a substitute for Phase I studies that further investigate safety and tolerability at multiple doses. Figure 2 summarizes the main differences between Phase 0 and Phase I trials. While the primary goal of a Phase I trial is to establish the maximum tolerated dose for a compound, a Phase 0 trial's primary aim is target modulation. As a consequence of this, dosing and dose escalation are limited in Phase 0 trials. Historical guidelines recommend that the starting dose for Phase I clinical trials in oncology, for example, be one-tenth of that dose which causes severe toxicity or death (STD) in $10 \%$ of animals - generally rodents - (onetenth the $\mathrm{STD}_{10}$; in $\mathrm{mg} / \mathrm{m}^{2}$ ), provided that this dose does not cause severe, irreversible toxicity in the other mammalian (non-rodent) species tested. ${ }^{13,14}$ The starting dose for Phase 0 trials with a PK or PD endpoint is generally 1/50th the rat "no observed adverse effect level" (NOAEL). For studies that do not focus on a PD endpoint, the dose selected should allow a substantial margin of safety (eg, a dose $100 \times$ higher did not cause toxicity in the single-dose toxicity study). Thus, the Phase 0 maximum dose can be that at which a PK/PD response is observed or target modulation is measured, as long as no drug-associated toxicity is found, and/or that the dose is less than one quarter of the rat NOAEL, or that the total exposure to drug measured in human blood samples (ie, area under the curve) up to half of that measured in the most sensitive species. ${ }^{8}$ At this point it is necessary to emphasize that a distinction has to be made between studies administering microdoses (such studies assess drug PK parameters: binding affinity and absorption; distribution; metabolism; and excretion) and those administering pharmacologically active but subtherapeutic doses (which assess more specific, predefined PK/PD endpoints). Moreover, because of the relatively small amount of study drug necessary to conduct a Phase 0 trial, there is no requirement for a full-scale CGMP (clinical good manufacturing practice)-grade commercial manufacturing prior to trial start. ${ }^{4}$

Due to the proof-of-concept nature of Phase 0 studies, the number of trial participants is smaller than that for Phase I, usually 10 to 15 subjects. This reduced sample size has further 


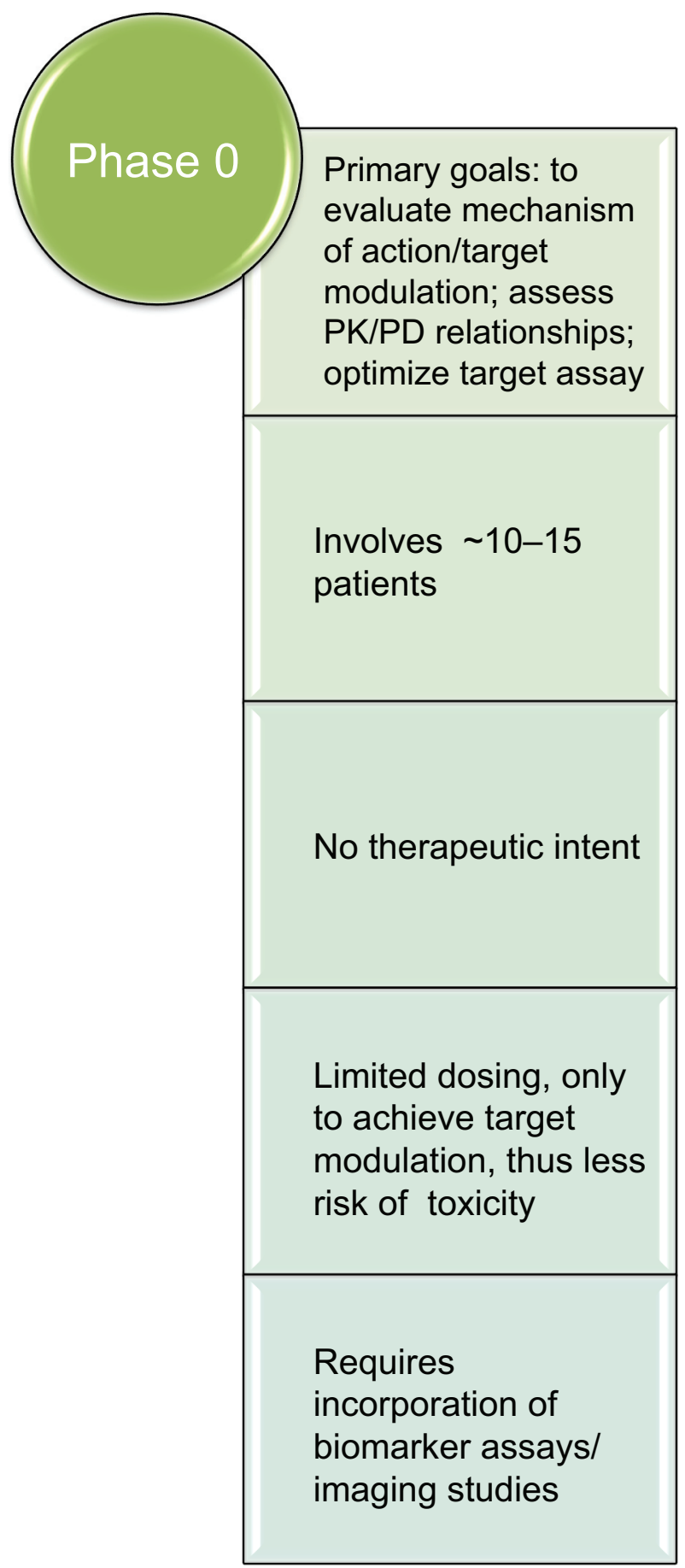

Figure 2 Phase 0 versus Phase I studies.

Abbreviations: PD, pharmacodynamic; PK, pharmacokinetic.

implications on the assessment of PK/PD endpoints insofar as the statistical analysis, choice of PD assay, and intra-patient/ inter-patient variability are concerned. With Phase 0 trials, patients are exposed to lower doses of the drug than in traditional Phase I trials, thus the associated risk of toxicity is likewise lower. While eligibility and profile of participants in Phase 0 and Phase I may not necessarily be different, ethical

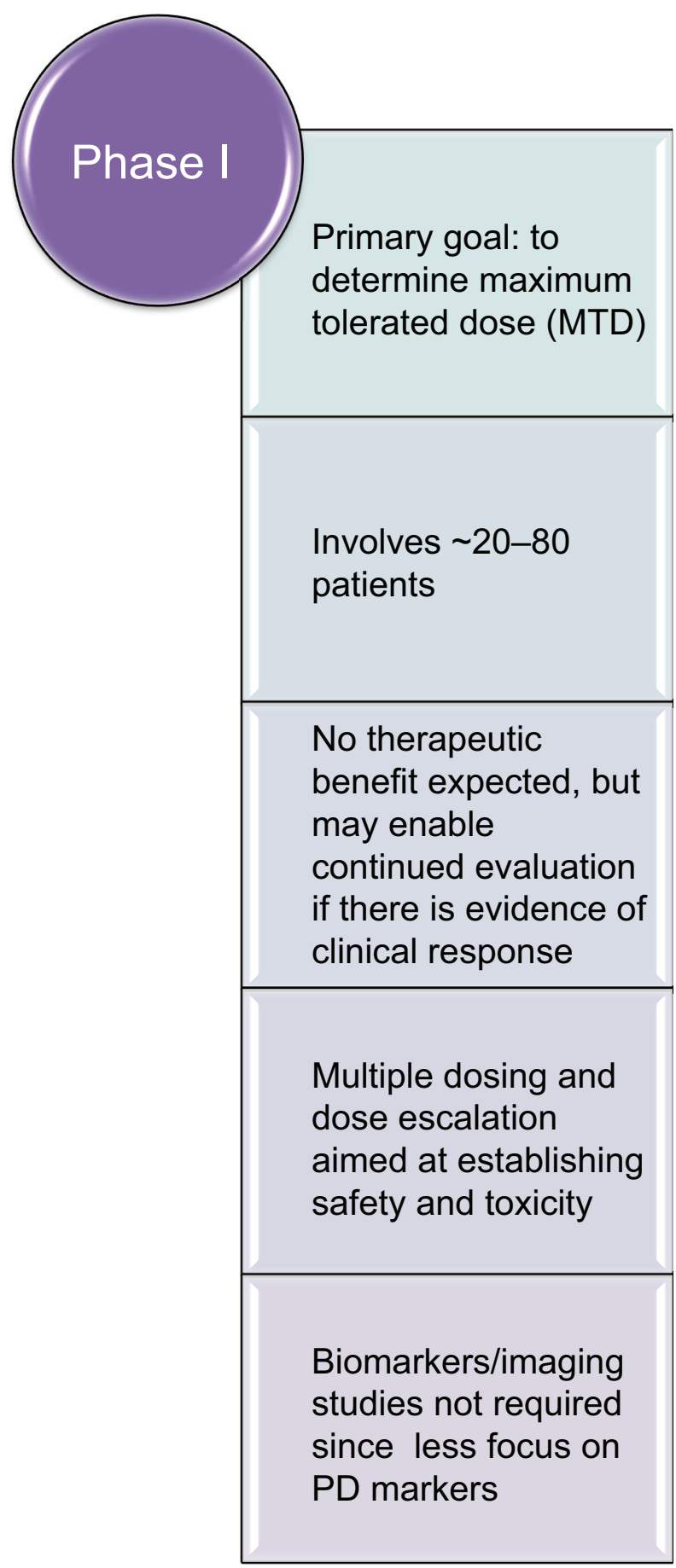

Biomarkers/imaging studies not required since less focus on PD markers

concerns regarding informed consent are certainly not the same due to the lack of therapeutic intent in Phase 0 studies. (Ethical considerations are discussed in another section.)

Phase 0 trials obviously have their merits, but not all novel agents are appropriate for Phase 0 testing. Phase 0 studies are intended to provide flexibility in the drug development process, particularly for drug and biological 
products meant to treat a serious or life-threatening illness. ${ }^{8}$ In the traditional Phase I trial for oncologic agents as well as other therapeutic areas, preliminary safety pharmacology studies are conducted to evaluate the systemic effects of a new agent on the cardiovascular system, central nervous system, and respiratory system; such studies can be conducted as part of later animal studies. Single-dose (acute) toxicology studies are required in two mammalian species to determine toxic and safe doses. In oncologic trials, detailed clinical observations following dosing and appropriate electrocardiographic measurements in non-rodents are generally considered sufficient. ${ }^{15}$ On the other hand, the US FDA may not require safety pharmacology studies for a Phase 0 trial if it involves a single microgram-quantity dose for imaging or PK analysis.

\section{Proof-of-concept}

The adenosine diphosphate (ADP)-ribose polymerase inhibitor ABT-888 (veliparib) is one of the first compounds that followed the Phase 0 trial paradigm. The Phase 0 trial was conducted by a team of investigators from Abbott Laboratories and the National Cancer Institute. ${ }^{16,17}$ Preclinical data showed that tumor poly (ADP-ribose) polymerase (PARP) inhibition was the target of ABT- 888 and a validated assay was available. Furthermore, at preclinical evaluation, efficacy of ABT- 888 was demonstrated at concentrations that inhibit tumor PARP but which do not markedly increase the agent's toxicity. Thus, it was reasonable to conduct a Phase 0 study that would be aimed at evaluating the mechanism of action at doses that posed minimal risk of toxicity to human subjects. ${ }^{16}$ The ABT-888 study enrolled 14 adult patients with advanced malignancies (or chronic lymphocytic leukemia and follicular lymphoma) refractory to at least one line of standard treatment. Patients with primary brain tumors, brain metastases, or a history of seizures were excluded because high-dose ABT- 888 was found to cause seizures in a preclinical animal model. Prior antineoplastic therapy must have been completed at $\geq 2$ weeks prior to study enrollment. ${ }^{17}$

ABT- 888 fit the profile of an ideal Phase 0 agent: the target could be monitored, the biomarker could be assayed with validity and reproducibility, and its PKs appeared linear. ${ }^{17,18}$ The ABT- 888 Phase 0 study addressed two fundamental questions that had to be satisfied in order to proceed with drug development: 1) whether or not the target plasma concentration is achievable with oral dosing; and 2) whether or not tumor biopsies are able to provide definitive results after a single dose of the investigational agent.
Another example - and at the other end of the spectrum is that of SR13668, an orally active AKT pathway inhibitor, which has demonstrated cancer chemopreventive potential in preclinical studies. The emerging field of chemoprevention agents encounters important barriers in development, including larger-scale clinical trials (such agents being typically intended for chronic use by healthy individuals), lengthy time frames between discovery and approval, liability risks (because they are given to healthy individuals), and a growing funding gap for early-stage candidates. ${ }^{19,20}$ Chemopreventive agents derived from dietary sources in particular provide an excellent opportunity for Phase 0 evaluation, because such agents need to have a relatively wide therapeutic window. AKT is an anti-apoptotic proto-oncogene whose overexpression is hypothesized to be an early event in carcinogenesis, based on immunohistochemical analyses detecting phosphoAKT in premalignant lung and colon lesions, with minimal or no expression in surrounding normal tissues. ${ }^{21,22} \mathrm{~A}$ Phase 0 chemoprevention trial enabled the rapid identification of a lead formulation of SR13668 for further clinical testing. Healthy adult volunteers were randomly assigned to receive a single oral dose of SR13668 in one of five different formulations (selection of the appropriate reference formulation was based on previous animal studies). Blood samples were obtained pre- and post-agent administration for PK analyses, with the area under the plasma concentration-time curve being the primary endpoint. ${ }^{23}$

The two novel compounds cited above show the feasibility of the Phase 0 paradigm, but earlier examples indicate that the idea behind Phase 0 studies has been around even before the US FDA (and EMA) guidance documents. ${ }^{24-28}$ It remains clear, however, that in Phase 0 trials, study design must integrate measures to quantify drug effect (eg, a PD assay of whether the agent inhibits a specific enzyme) to allow rational decisions about further drug development. ${ }^{29}$ It is important to note that the presence of a validated assay for experimental drug activity should not be misconstrued as presence of a validated biomarker.

Two other areas where Phase 0 trials may prove invaluable for drug development are studies combining molecularly targeted drugs and for clinical trials of molecular imaging agents. The opportunity to administer two or more experimental or US FDA-approved drugs while collecting appropriate PK data will contribute significantly to a better understanding of the bioavailability of these agents. The potential to correlate PK and PD data allows further assessment and substantiation of any synergistic activity with minimal risk to patients from combination toxicity; 
hence, optimal relative doses and dosing schedules can be determined prior to toxicology and tolerability assessment in Phase I testing. ${ }^{11}$

\section{Ethical considerations}

In an earlier editorial discussing the ethics of Phase 0 trials, Hill emphasized that while both scientific validity and benefit to participants are necessary conditions justifying participation of human subjects in clinical research, only benefit (but not scientific validity) is considered a sufficient condition. ${ }^{30}$ The term benefit in clinical research can mean benefit that is direct, indirect, or "to others." Given the design and purpose of Phase 0 trials, it seems that "benefit to others" is the singular possible benefit expected, underlining the ethical challenge that such trials are up against, particularly with respect to the fundamental principle that the interests of human subjects must always take precedence over the interests of society. ${ }^{31}$ Since Phase 0 trials will definitely not benefit the enrolled person, the standard of information disclosure is more stringent, conforming to the "reasonable volunteer" standard stated in the Belmont Report on Ethical Principles and Guidelines for Research Involving Human Subjects. The "reasonable volunteer standard" is where the nature and amount of information is enough to enable the persons volunteering for research to know that the trial intervention is neither necessary for their care, nor is the intervention fully understood as a form of therapy, so that they can decide whether they want to participate or not. ${ }^{32}$ The participant should have no "false hopes" of the trial intervention working for the benefit of the participant; that is, therapeutic misconception is a valid point, which needs to be made clear to the participant.

Phase 0 trials involving healthy volunteers can also be considered, from an ethical point of view, a special case of the healthy volunteer studies of Phase I. What seems to be more controversial is the issue of studies aimed at establishing dose and proof of concept in the setting of oncologic drugs. Most Phase 0 oncology trial participants are likely to be drawn from the same population as Phase I subjects, and while they indeed comprise a vulnerable population, these patients are capable of understanding and appreciating information and expressing voluntary preferences in the context of non-beneficial research with more-thanminimal risk. ${ }^{33-35}$ Experience with the ABT-888 Phase 0 trial showed that patients and patient advocates are generally supportive of efforts to speed up the drug development process. ${ }^{36}$ Nevertheless, there can be situations described as confusing "aspiration with self-interest", where prospective trial subjects having the disease of interest believe that participating in the study will advance the development of drugs that they might later receive; ${ }^{37}$ investigators should be clear about this issue at the outset. Investigators need to be forthright in discussing with prospective participants the nontherapeutic nature of Phase 0 trials, the purpose of such trials, as well as the potential implications on the development of the investigational drug. Informed consent for study participants should make explicit that the dose of the investigational agent to be administered is lower than that which would be expected to lead to therapeutic benefit - or cause appreciable toxicity.

This ethical constraint of providing a microdose intervention with no anticipated clinical benefits is much less challenging in chemoprevention trials, which generally exclude patients with cancer or other unstable medical conditions. ${ }^{23}$ Thus, Phase 0 trials can bring opportunities to accelerate chemoprevention agent development under this novel paradigm. ${ }^{38}$ Because fewer toxicologic data are required for Phase 0 trials, there is also concern that such limited toxicology might be insufficient and may compromise patient safety, despite the very low dose and limited duration of exposure. It remains essential that all patients be closely monitored for any side effects, especially for oncologic agents, which are all potentially toxic. Moreover, evaluation of response to the agent often requires invasive procedures such as tissue biopsy (which may also have to be done more than once). Repeated assessments may be misconstrued by some as an indication of therapeutic benefit; the intent of such procedures should be made clear to patients.

Another concern for patients is the possible delay - or even exclusion - from future participation in certain clinical trials. While the duration of participation in a Phase 0 trial is expected to be short because of limited dosing schedules ( $\sim 7-14$ days), this issue should be discussed with the patient. In addition, Phase 0 trials should be considered only for those patients who do not have symptoms that require immediate therapy. It is imperative that investigators advocate for Phase 0 trial participants to be subsequently treated - either with conventional treatment or on another clinical trial.

Indeed, it can be said that Phase 0 (cancer) trials are both ethically challenged and ethically challenging. ${ }^{30}$ The issue of being ethically challenged can be overcome with scientifically valid and rigorous methodology; the issue of being ethically challenging remains a hurdle in the sense that the best argument would be to say that there is a moral obligation to participate in clinical research (which remains controversial). The closer investigators are to satisfying these 
two conditions, the closer society at-large is to accept the Phase 0 trial paradigm.

\section{Summary and perspectives}

Who will ultimately benefit from Phase 0 clinical trials? And are Phase 0 trials really necessary $?^{27}$ As far as the US FDA and the EMA are concerned, (the introduction of the concept of) Phase 0 trials was necessary because the existing paradigm of drug development was based on the assumption of an investigational compound's toxicity being a function of dose as well as the idea of efficacy being somewhat related to toxicity. ${ }^{39}$ It has been approximately 10 years since the introduction of the concept of Phase 0 trials (can be more or less, depending on the point of reference), but it will take some time before it can be concluded whether Phase 0 trials have a positive impact on the development of new drugs for cancer or other indications, independent of whether exploratory IND studies result in further development of promising candidate products or elimination of nonviable agents. The ABT- 888 and SR13668 trials cited earlier are important examples resulting in the further development of these agents and can help assess the added value of the exploratory IND approach to the drug development armamentarium. A survey conducted within the Pharmaceutical Research and Manufacturers of America, published in 2010, revealed that while the pharmaceutical industry is still taking a circumspect approach to the Phase 0 paradigm, the potential usefulness for early clinical guidance in drug development is recognized. ${ }^{40}$ It is anticipated that PD-driven studies will expedite the evaluation of those agents that directly modulate their targets. Patient safety remains paramount, but the emphasis of Phase 0 first-in-human testing is on a drug's target rather than its toxicity.

Because drugs that fail proof-of-principle target inhibition studies may be discarded before reaching formal Phase I/II evaluation, a potential drawback of using a Phase 0 trial to eliminate nonviable candidate products is the premature dismissal of a promising candidate. This may occur for instance when the PKs of a microdose (as evaluated in a Phase 0 study) does not have good correlation with the therapeutically relevant dose. ${ }^{11}$ Understanding of tumorigenesis and of the mechanism of targeted therapies remains inadequate, and so this issue may be a particularly important concern in oncologic drug development. The lack of reliable and validated assays (which are not readily available even for most approved targeted cancer drugs) could result in mistakenly classifying active drugs as inactive. ${ }^{41}$

A Phase 0 study is thus more valuable when considered from the point of view of a discovery, rather than a development tool. ${ }^{18}$ While the motivation behind Phase 0 trials/exploratory IND is to accelerate the process of drug development by providing opportunities to streamline target identification/modulation and refine the lead optimization process in vivo, promising drugs will still need to be further evaluated for toxicity and efficacy under a traditional IND. Under the appropriate circumstances, Phase 0 trials may help to eliminate drugs that are likely to fail later-stage efficacy testing well before moving into trials that require large numbers of patients to establish drug tolerability and safety. The goals of this effort are to identify promising agents earlier, develop and establish PD assays in human samples prior to instituting larger trials, and potentially shorten the drug development timeline. Accomplishing these goals may then increase the success rate of new agents entering clinical development and bring active drugs to market faster.

Important factors that need to be considered when designing Phase 0 trials include: 1) evidence for linear PKs of the candidate drug; 2) availability of a sensitive bioassay; 3 ) adequate infrastructure and dedicated and qualified research team; 4) availability of a measurable PD effect at very low doses; 5) feasibility of tumor tissue sampling vis-àvis ethical considerations; and 6) availability of appropriate trial subjects.

\section{Disclosure}

The author reports no conflicts of interest in this work.

\section{References}

1. DiMasi JA, Hansen RW, Grabowski HG. The price of innovation: new estimates of drug development costs. $J$ Health Econ. 2003;22(2): 151-185.

2. Kaitin KI. Deconstructing the drug development process: the new face of innovation. Clin Pharmacol Ther. 2010;87(3):356-361.

3. Ledford H. Translational research: 4 ways to fix the clinical trial. Nature. 2011;477(7366):526-528.

4. Kummar S, Rubinstein L, Kinders R, et al. Phase 0 clinical trials: conceptions and misconceptions. Cancer J. 2008;14(3):133-137.

5. FDA Guidance for Industry. Animal Models - Essential Elements to Address Efficacy Under the Animal Rule. Silver Spring, MD: US Food and Drug Administration. Available from: http://www.fda. gov/downloads/Drugs/GuidanceComplianceRegulatoryInformation/ Guidances/ucm078923.pdf. Accessed on September 24, 2013.

6. Owens J. Determining druggability. Nat Rev Drug Discov. 2007; 6:187.

7. US Department of Health and Human Services. Food and Drug Administration. Challenge and Opportunity on the Critical Path to New Medical Products. 2004. Available from: http://www.fda.gov/downloads/ ScienceResearch/SpecialTopics/CriticalPathInitiative/CriticalPathOppo rtunitiesReports/ucm113411.pdf. Accessed September 24, 2013.

8. US Department of Health and Human Services. Food and Drug Administration, Center for Drug Evaluation and Research (CDER). Guidance for Industry, Investigators, and Reviewers - Exploratory IND Studies. 2006. Available from: http://www.fda.gov/downloads/Drugs/Guida nceComplianceRegulatoryInformation/Guidances/UCM078933.pdf. Accessed September 24, 2013. 
9. European Medicines Agency (EMA). Concept Paper on the Development of a CHMP Guideline on the Non-clinical Requirements to Support Early Phase I Clinical Trials with Pharmaceutical Compounds. London: EMA; 2006. Available from: http://www.ema.europa.eu/docs/en_GB/ document_library/Scientific_guideline/2009/10/WC500003979.pdf. Accessed September 4, 2013.

10. European Medicines Agency. ICH guideline M3(R2) on non-clinical safety studies for the conduct of human clinical trials and marketing authorisation for pharmaceuticals. Available from: http://www.ema. europa.eu/docs/en_GB/document_library/Scientific_guideline/2009/09/ WC500002720.pdf. Accessed September 4, 2013.

11. Marchetti S, Schellens JH. The impact of FDA and EMEA guidelines on drug development in relation to Phase 0 trials. $\mathrm{Br} J$ Cancer. 2007;97(5):577-581.

12. Kinders R, Parchment RE, Ji J, et al. Phase 0 clinical trials in cancer drug development: from FDA guidance to clinical practice. Mol Interv. 2007;7(6):325-334.

13. Kummar S, Kinders R, Rubinstein L, et al. Compressing drug development timelines in oncology using phase ' 0 ' trials. Nat Rev Cancer. 2007;7(2):131-139.

14. DeGeorge JJ, Ahn CH, Andrews PA, et al. Regulatory considerations for preclinical development of anticancer drugs. Cancer Chemother Pharmacol. 1998;41(3):173-185.

15. FDA. Guidance for Industry: 99 Nonclinical Evaluation for Anticancer Pharmaceuticals. Available from: http://www.fda. gov/downloads/Drugs/.../Guidances/ucm085389.pdf. Accessed September 4, 2013.

16. Eliopoulos H, Giranda V, Carr R, Tiehen R, Leahy T, Gordon G. Phase 0 trials: an industry perspective. Clin Cancer Res. 2008;14(12): 3683-3688.

17. Kummar S, Kinders R, Gutierrez ME, et al. Phase 0 clinical trial of the poly (ADP-ribose) polymerase inhibitor ABT-888 in patients with advanced malignancies. J Clin Oncol. 2009;27(16): 2705-2711.

18. LoRusso PM. Phase 0 clinical trials: an answer to drug development stagnation? J Clin Oncol. 2009;27(16):2586-2588.

19. Herberman RB, Pearce HL, Lippman SM, Pyenson BS, Alberts DS. Cancer chemoprevention and cancer preventive vaccines - a call to action: leaders of diverse stakeholder groups present strategies for overcoming multiple barriers to meet an urgent need. Cancer Res. 2006;66(24):11540-11549.

20. Grabowski HG, Moe JL. Impact of economic, regulatory, and patent policies on innovation in cancer chemoprevention. Cancer Prev Res (Phila). 2008;1(2):84-90.

21. Roy HK, Olusola BF, Clemens DL, et al. AKT proto-oncogene overexpression is an early event during sporadic colon carcinogenesis. Carcinogenesis. 2002;23(1):201-205.

22. Balsara BR, Pei J, Mitsuuchi Y, et al. Frequent activation of AKT in non-small cell lung carcinomas and preneoplastic bronchial lesions. Carcinogenesis. 2004;25(11):2053-2059.
23. Reid JM, Walden CA, Qin R, et al. Phase 0 clinical chemoprevention trial of the Akt inhibitor SR13668. Cancer Prev Res (Phila). 2011;4(3): 347-353.

24. Garner RC, Lappin G. The phase 0 microdosing concept. Br J Clin Pharmacol. 2006;61(4):367-370.

25. Saleem A, Harte RJ, Matthews JC, et al. Pharmacokinetic evaluation of $\mathrm{N}$-[2-(dimethylamino)ethyl]acridine-4-carboxamide in patients by positron emission tomography. J Clin Oncol. 2001;19(5):1421-1429.

26. Meerum Terwogt JM, Groenewegen G, Pluim D, et al. Phase I and pharmacokinetic study of SPI-77, a liposomal encapsulated dosage form of cisplatin. Cancer Chemother Pharmacol. 2002;49(3):201-210.

27. Camporesi S. Phase 0 Workshop at the 20th EORTC-NCI-AACR Symposium, Geneva. Ecancermedicalscience. 2008;2:107.

28. Lappin G, Kuhnz W, Jochemsen R, et al. Use of microdosing to predict pharmacokinetics at the therapeutic dose: experience with 5 drugs. Clin Pharmacol Ther. 2006;80(3):203-215.

29. Findlay JW, Smith WC, Lee JW, et al. Validation of immunoassays for bioanalysis: a pharmaceutical industry perspective. J Pharm Biomed Anal. 2000;21(6):1249-1273.

30. Hill TP. Phase 0 trials: are they ethically challenged? Clin Cancer Res. 2007;13(3):783-784.

31. WMA.net [homepage on the Internet]. WMA Declaration of Helsinki - ethical principles for medical research involving human subjects. France: World Medical Association; 2008. Available from: http://www.wma.net/en/30publications/10policies/b3. Accessed April 10, 2013.

32. The Belmont Report. Ethical Principles and Guidelines for the Protection of Human Subjects of Research. Available from: http://www.fda. gov/ohrms/dockets/ac/05/briefing/2005-4178b_09_02_Belmont $\% 20$ Report.pdf. Accessed September 4, 2013.

33. Agrawal M, Emanuel EJ. Ethics of phase 1 oncology studies: reexamining the arguments and data. JAMA. 2003;290(8):1075-1082.

34. Agrawal M. Voluntariness in clinical research at the end of life. J Pain Symptom Manage. 2003;25(4):S25-S32.

35. Abdoler E, Taylor H, Wendler D. The ethics of phase 0 oncology trials. Clin Cancer Res. 2008;14(12):3692-3697.

36. Gutierrez M, Collyar D. Patient perspectives on phase 0 clinical trials. Clin Cancer Res. 2008;14(12):3689-3691.

37. Kimmelman J. Ethics at phase 0: clarifying the issues. J Law Med Ethics. 2007;35(4):727-733, 514.

38. Murgo AJ, Kummar S, Rubinstein L, et al. Designing phase 0 cancer clinical trials. Clin Cancer Res. 2008;14(12):3675-3682.

39. Fox E, Curt GA, Balis FM. Clinical trial design for target-based therapy. Oncologist. 2002;7(5):401-409.

40. Karara AH, Edeki T, McLeod J, Tonelli AP, Wagner JA. PhRMA survey on the conduct of first-in-human clinical trials under exploratory investigational new drug applications. J Clin Pharmacol. 2010;50(4): 380-391.

41. Twombly R. Slow start to phase 0 as researchers debate value. $J$ Natl Cancer Inst. 2006;98(12):804-806.
Open Access Journal of Clinical Trials

\section{Publish your work in this journal}

The Open Access Journal of Clinical Trials is an international, peerreviewed, open access journal publishing original research, reports, editorials, reviews and commentaries on all aspects of clinical trial design, management, legal, ethical and regulatory issues, case record form design, data collection, quality assurance and data auditing
Dovepress

methodologies. The manuscript management system is completely online and includes a very quick and fair peer-review system, which is all easy to use. Visit http://www.dovepress.com/testimonials.php to read real quotes from published authors. 